\title{
Implementation of maternal and perinatal deaths surveillance and response system among health facilities in Rural Tanzania: Analytical cross- sectional study
}

Christina Kashililika

University of Dodoma

Fabiola Vincent Moshi ( $\sim$ fabiola.moshi@gmail.com )

University of Dodoma

\section{Research Article}

Keywords: MPDSR, maternal death, perinatal death

Posted Date: March 4th, 2021

DOI: https://doi.org/10.21203/rs.3.rs-181872/v1

License: (c) (i) This work is licensed under a Creative Commons Attribution 4.0 International License. Read Full License 


\section{Abstract}

\section{Background}

Maternal and Perinatal Deaths Review and Surveillance (MPDSR) system when used effectively has the power to bring into reality, a revolutionary victory in the fight against maternal and perinatal mortality from avoidable causes. This study aimed at determining the status of implementation of the system among health facilities in rural settings of Tanzania.

\section{Method}

This study was conducted among 38 health facilities from three districts of Morogoro region, Tanzania from April 27, 2020 to May 29, 2020. Quantitative data was collected through document review for MPDSR implementation status. The outcome was determined by using special scoring sheet with a total 30 points. Facilities that scored 10 points or above were considered to have satisfactory status of MPDSR implementation while the facilities that scored below 10 points were considered to have unsatisfactory status of MPDSR implementation. Bivariate logistic regression analyses were used to determine the predictors of implementation status among health facilities.

\section{Results}

Majority of health facilities $20(52.6 \%)$ had satisfactory MPDSR implementation status. The predictors of MPDSR implementation in a facility were level of health facility [Hospital $(A O R=11.945$ at $95 \% \mathrm{Cl}=$ $1.133-125.942, P=0.039)$ ] and ownership of the facility [Public (AOR $=0.133$ at $95 \% \mathrm{Cl}=0.019-$ $0.920, P=0.041)]$.

\section{Conclusion}

MPDSR implementation status among health facility is on average not satisfactory. More efforts are needed to raise the status of MPDSR implementation in the country so that the maximum benefit of MPDSR is obtained.

\section{Full Text}

Due to technical limitations, full-text HTML conversion of this manuscript could not be completed. However, the latest manuscript can be downloaded and accessed as a PDF.

\section{Tables}

\section{Table 1: Facility Characteristics $(n=38)$}




\begin{tabular}{lll}
\hline Variable & Frequency (n) & Percentage (\%) \\
\hline Level & & \\
Hospital & 11 & 28.9 \\
\hline Health Centre & 27 & 71.1 \\
\hline Location & & \\
\hline Urban & 10 & 26.3 \\
\hline Rural & 28 & 73.7 \\
\hline Ownership & & \\
\hline Public & 25 & 65.8 \\
\hline Private & 13 & 34.2 \\
\hline
\end{tabular}

Table 2: MPDSR tools and protocols $(\mathrm{n}=38)$

\begin{tabular}{|c|c|c|}
\hline Item & $\begin{array}{l}\text { YES n } \\
(\%)\end{array}$ & $\begin{array}{l}\text { NO n } \\
(\%)\end{array}$ \\
\hline $\begin{array}{l}\text { There are written policies, guidelines or protocols regarding the } \\
\text { practice of MPDSR }\end{array}$ & $\begin{array}{l}11 \\
(28.9)\end{array}$ & $\begin{array}{l}27 \\
(71.1)\end{array}$ \\
\hline Data collection forms are available & $\begin{array}{l}37 \\
(97.4)\end{array}$ & $1(2.6)$ \\
\hline Tools include causes of deaths & $\begin{array}{l}35 \\
(92.1)\end{array}$ & $3(7.9)$ \\
\hline Tools include modifiable factors for the cause of death & $\begin{array}{l}35 \\
(92.1)\end{array}$ & $3(7.9)$ \\
\hline Tools include place to follow up on actions taken & $3(7.9)$ & $\begin{array}{l}35 \\
(92.1)\end{array}$ \\
\hline Attendance is mandatory & $\begin{array}{l}20 \\
(52.6)\end{array}$ & $\begin{array}{l}18 \\
(47.4)\end{array}$ \\
\hline Death review meeting are held at stated interval & $1(2.6)$ & $\begin{array}{l}37 \\
(97.4)\end{array}$ \\
\hline Data trends is displayed or shared & $0(0.0)$ & $\begin{array}{l}38 \\
(100.0)\end{array}$ \\
\hline $\begin{array}{l}\text { Evidence of change based on recommendation arising from death } \\
\text { review findings }\end{array}$ & $0(0.0)$ & $\begin{array}{l}38 \\
(100.0)\end{array}$ \\
\hline
\end{tabular}


Table 3: Staff involvement in MPDSR system $(\mathrm{n}=38)$

\begin{tabular}{|c|c|c|}
\hline Item & $\begin{array}{l}\text { YES n } \\
(\%)\end{array}$ & $\begin{array}{l}\text { NO n } \\
(\%)\end{array}$ \\
\hline $\begin{array}{l}\text { Special persons who take specific effort in promoting death reviews } \\
\text { including management, professionals, driving forces }\end{array}$ & $\begin{array}{l}38 \\
(100.0)\end{array}$ & $0(0.0)$ \\
\hline $\begin{array}{l}\text { The coordinator(s) have other responsibilities (e.g. information officer, } \\
\text { QI focal point, etc.) }\end{array}$ & $\begin{array}{l}38 \\
(100.0)\end{array}$ & $0(0.0)$ \\
\hline Clear leader(s) involved in establishing and championing death reviews & $\begin{array}{l}36 \\
(94.7)\end{array}$ & $2(5.3)$ \\
\hline $\begin{array}{l}\text { Has anyone in facility or district leadership signed a commitment or } \\
\text { undertaken an agreement that s/he would ensure that MPDSR is } \\
\text { implemented in the facility? }\end{array}$ & $0(0.0)$ & $\begin{array}{l}38 \\
(100.0)\end{array}$ \\
\hline Facility in charge chairs the MPDSR meeting & $\begin{array}{l}21 \\
(55.3)\end{array}$ & $\begin{array}{l}17( \\
44.7)\end{array}$ \\
\hline Evidence that staff have received MPDSR & $0(0.0)$ & $\begin{array}{l}38 \\
(100.0)\end{array}$ \\
\hline
\end{tabular}

Table 4: Relationship between facility characteristics and level of MPDSR implementation

\begin{tabular}{lllll}
\hline Variable & Satisfactory $\mathbf{n ~ ( \% ) ~}$ & Unsatisfactory n (\%) & $\mathrm{X}^{2}$ & P value \\
\hline $\begin{array}{c}\text { Location } \\
\text { Urban }\end{array}$ & $6(60.0)$ & $4(40.0)$ & & \\
\hline Rural & $12(42.9)$ & $16(51.1)$ & 0.869 & 0.351 \\
\hline Level & & & & \\
\hline Hospital & $10(90.9)$ & $1(9.1)$ & 11.77 & 0.001 \\
\hline Health center & $8(29.6)$ & $19(70.4)$ & & \\
Ownership & & & & \\
\hline Public & $7(28.0)$ & $18(72.0)$ & 10.10 & 0.001 \\
\hline Private & $11(84.6)$ & $2(15.4)$ & &
\end{tabular}

Table 5: Association between facility characteristics and status of MPDSR implementation 


\begin{tabular}{|c|c|c|c|c|c|c|c|c|}
\hline \multirow[b]{2}{*}{ Variable } & \multirow[b]{2}{*}{ OR } & \multicolumn{2}{|c|}{$95 \% \mathrm{CI}$} & \multirow[t]{2}{*}{ P-value } & \multirow[b]{2}{*}{ AOR } & \multicolumn{2}{|c|}{$95 \% \mathrm{CI}$} & \multirow[t]{2}{*}{ P-value } \\
\hline & & Lower & Upper & & & Lower & Upper & \\
\hline \multicolumn{9}{|c|}{ Location of facility } \\
\hline Rural & 1 & & & & 1 & & & \\
\hline Urban & 2.000 & 0.460 & 8.698 & 0.355 & 1.810 & 0.269 & 12.18 & 0.542 \\
\hline \multicolumn{9}{|l|}{ Level of facility } \\
\hline Health center & 1 & & & & & & & \\
\hline Hospital & 23.75 & 2.591 & 217.67 & 0.005 & 11.94 & 1.133 & 125.94 & 0.039 \\
\hline \multicolumn{9}{|l|}{ Ownership } \\
\hline Private & 1 & & & & & & & \\
\hline Public & 0.071 & 0.012 & 0.403 & 0.003 & 0.133 & 0.019 & 0.920 & 0.041 \\
\hline
\end{tabular}

\section{Figures}

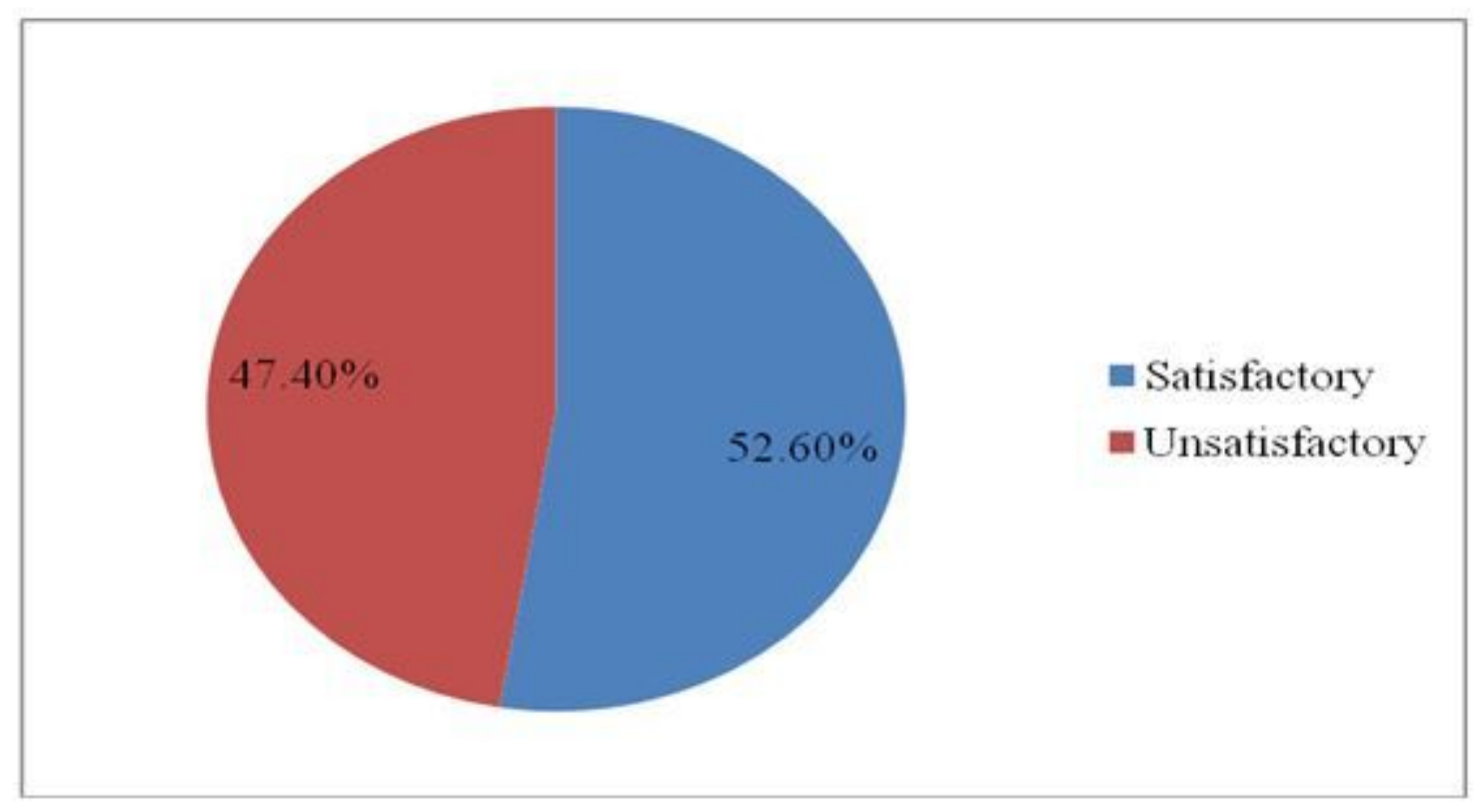

Figure 1

status of MPDSR implementation among health facilities $(n=38)$ 


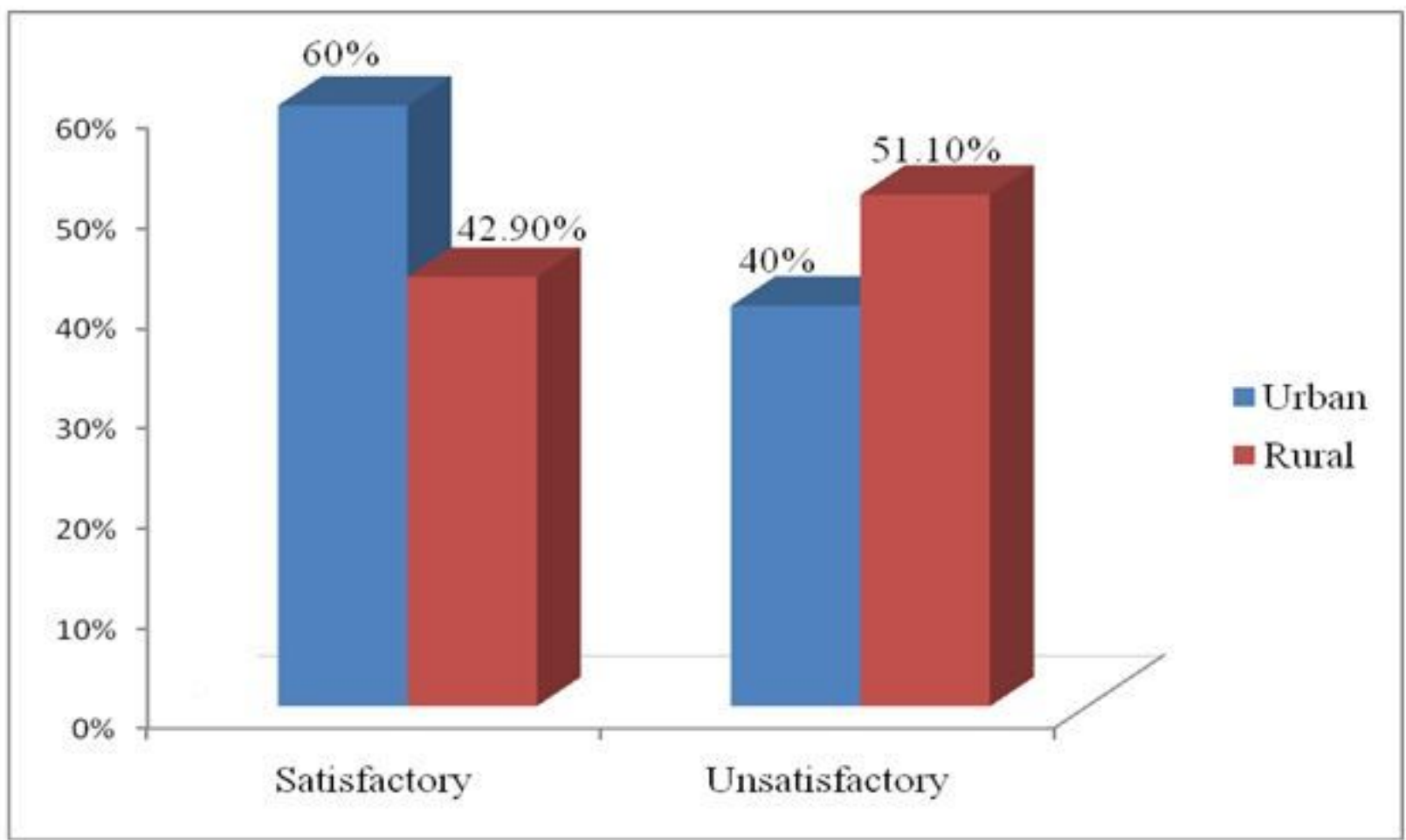

Figure 2

MPDSR implementation status among health facilities according to their location in Morogoro region 